\title{
Survival Estimates after Stopping Sorafenib in Patients with Hepatocellular Carcinoma: NEXT Score Development and Validation
}

Hye Won Lee ${ }^{1}$, Hyun Soo Kim ${ }^{1}$, Seung Up Kim ${ }^{1,2,3}$, Do Young Kim ${ }^{1,2,3}$, Beom Kyung Kim ${ }^{1,2,3}$, Jun Yong Park ${ }^{1,2,3}$, Sang Hoon Ahn ${ }^{1,2,3}$, Mi Young Jeon ${ }^{1}$, Ja Yoon Heo ${ }^{1}$, Soo Young Park ${ }^{4}$, Yu Rim Lee ${ }^{4}$, Sun Kyung Jang ${ }^{4}$, Su Hyun Lee ${ }^{4}$, Se Young Jang ${ }^{4}$, Won Young Tak ${ }^{4}$, and Kwang-Hyub Han ${ }^{1,2,3}$

${ }^{1}$ Department of Internal Medicine, ${ }^{2}$ Institute of Gastroenterology, Yonsei University College of Medicine, ${ }^{3}$ Liver Cirrhosis Clinical Research Center, Seoul, and ${ }^{4}$ Department of Internal Medicine, Kyungpook National University School of Medicine, Daegu, Korea

Background/Aims: Limited information is available regarding patient survival after sorafenib discontinuation in patients with hepatocellular carcinoma (HCC). Thus, we developed and validated a novel survival prediction model. Methods: Clinical data from 409 patients with HCC who stopped taking sorafenib between September 2008 and February 2015 were reviewed. Results: In the training cohort, four factors were independent negative predictors of survival $(p<0.05)$. Based on the $\beta$ regression coefficient of each factor, we established the NEXT score (Survival after Stopping Nexavar Treatment), allocating 1 point each for an Eastern Cooperative Oncology Group score $\geq 2$, Child-Pugh class B or C, serum sodium $\leq 135 \mathrm{mEq} / \mathrm{L}$, and $\alpha$-fetoprotein $>400 \mathrm{ng} / \mathrm{mL}$. Area under the receiver operating characteristic curve values to predict 1-, 3-, and 6-month survival rates were 0.805, 0.809, and 0.774 , respectively, in the training cohort and 0.783 , 0.728 , and 0.673 , respectively, in the validation cohort $(n=137)$. When the training and validation cohorts were stratified into three risk groups (NEXT score 0 [low-risk] vs 1 to 2 [intermediate-risk] vs 3 to 4 [high-risk]), survival differed significantly between the groups $(p<0.05$, log-rank test). Conclusions: In patients with HCC, survival after stopping sorafenib is poor. However, risk estimates based on a new "NEXT score" may help predict survival and prognosis even in patients who discontinue sorafenib treatment. (Gut Liver 2017;11:693-701)

Key Words: Hepatocellular carcinoma; Survival; Sorafenib; Prediction; Risk

\section{INTRODUCTION}

Hepatocellular carcinoma (HCC) is a major health problem, accounting for more than 700,000 new diagnoses per year. ${ }^{1}$ HCC is also the third most common cause of cancer-related deaths globally, after lung and stomach cancers. ${ }^{2}$ Although various therapeutic measures, such as surgery, local ablation, or transcatheter arterial chemoembolization, are often performed in patients with early or intermediate HCC, ${ }^{1}$ systemic chemotherapy for advanced HCC has yet to be established.

Sorafenib (Nexavar; Bayer HealthCare Pharmaceuticals, Whippany, NJ, USA; Onyx Pharmaceuticals, South San Francisco, CA, USA) is an orally active multi-kinase inhibitor that suppresses malignant cellular proliferation and neoplastic angiogenesis and increases the rate of apoptosis in a wide range of tumor models. ${ }^{3,4}$ In a clinical setting, both the Sorafenib Hepatocellular Carcinoma Assessment Randomized Protocol (SHARP) trial and the Asia-Pacific (AP) trial proved its efficacy, documenting prolonged median survival and a nearly 3-month extension of time to radiologic progression. ${ }^{5,6}$ Sorafenib is the only approved systemic targeted chemotherapy for patients with advanced unresectable HCC. ${ }^{7-10}$ Unfortunately, no proven second-line therapy is available to date, leaving supportive care or clinical trials of investigational molecular target agents as the only remaining options for patients who have failed guidelinestipulated sorafenib treatment. ${ }^{7}$

Most patients with advanced HCC experience disease progression or intolerability to sorafenib, even if liver function and performance status are acceptable. Although data on efficacy, safety, and prognosis of sorafenib use are available, ${ }^{11,12}$ little

\footnotetext{
Correspondence to: Seung Up Kim ${ }^{\mathrm{a}}$ and Do Young $\mathrm{Kim}^{\mathrm{b}}$

Department of Internal Medicine, Yonsei University College of Medicine, 50-1 Yonsei-ro, Seodaemun-gu, Seoul 03722, Korea

${ }^{a}$ Tel: +82-2-2228-1982, Fax: +82-2-393-6884, E-mail: ksukorea@yuhs.ac

bel: +82-2-2228-1992, Fax: +82-2-393-6884, E-mail: dyk1025@yuhs.ac

Received on August 5, 2016. Revised on November 28, 2016. Accepted on January 10, 2017. Published online June 27, 2017

pISSN 1976-2283 eISSN 2005-1212 https://doi.org/10.5009/gnl16391

Hye Won Lee and Hyun Soo Kim contributed equally to this work as first authors.

(a) This is an Open Access article distributed under the terms of the Creative Commons Attribution Non-Commercial License (http://creativecommons.org/licenses/by-nc/4.0) which permits unrestricted non-commercial use, distribution, and reproduction in any medium, provided the original work is properly cited.
} 
is known regarding the clinical disease course after treatment is discontinued. In a recent clinical trial where brivanib was used as a second-line therapy in patients who failed sorafenib therapy, the median survival time in the placebo arm was 8.2 months, ${ }^{13}$ which is similar to the survival time in the SHARP trial and even longer than that in the AP trial. This suggests that there is a wide range of patient survival rates, even if sorafenib is withdrawn. Consequently, it is important to predict survival and identify prognostic factors following cessation of sorafenib due to progressive disease or intolerability, thereby optimizing candidate selection for second-line anticancer treatments or for best supportive care. Recent studies have addressed this issue and proposed that post-sorafenib survival can be influenced by performance status, prothrombin time, extrahepatic tumor spread, macrovascular invasion, reason for sorafenib discontinuation, and type of progression (intrahepatic/extrahepatic increase in tumor size, new intrahepatic lesion, or new extrahepatic lesion). ${ }^{14,15}$

The aim of this retrospective study was to investigate survival outcomes and prognostic factors after sorafenib failure. In addition, a novel survival prediction model was developed and validated, using the prognostic factors identified.

\section{MATERIALS AND METHODS}

\section{Patients}

A total of 666 eligible patients with HCC who discontinued sorafenib treatment because of progressive disease $(n=292$, $43.8 \%$ ), intolerability due to adverse events ( $n=147,22.1 \%)$ and decreased liver function $(\mathrm{n}=167,25.1 \%)$, and noncompliance $(n=60,9.0 \%)$ between September 2008 and February 2015 were selected for the study from the database of Yonsei Liver Center (Seoul, Korea). HCC was diagnosed based on histologic or radiologic evaluation, in agreement with guidelines of the American Association for the Study of Liver Disease or the European Association for the Study of the Liver. ${ }^{16}$ Exclusion criteria were as follows: insufficient clinical data for statistical analysis, sorafenib treatment duration $<2$ weeks, other significant comorbidities which might influence performance status or survival, and obscure date of stopping sorafenib.

Our study protocol upheld ethical guidelines of the 1975 Declaration of Helsinki and was approved by the Institutional Review Board of Severance Hospital. Given the retrospective nature of this study, informed consent was not required.

\section{Sorafenib treatment}

Customary sorafenib dosage was $400 \mathrm{mg}$, twice daily. After initiating sorafenib treatment, all of the patients were monitored every 4 to 8 weeks by an experienced hepatologist with knowledge of the Common Terminology Criteria for Adverse Events (CTCAE). Sorafenib was administered continuously until disease progression based on the modified Response Evaluation Criteria in Solid Tumors (mRECIST) or early termination for grade 4 adverse events, decreased liver function, or noncompliance. During sorafenib treatment, if patients experienced grade 3 adverse events, the sorafenib dose was modified on an individual basis. After discontinuation of sorafenib for any reason, other options, such as clinical study participation or conventional systemic cytotoxic chemotherapy, were permissible as allowed by patient status and physician consent.

\section{Study protocol}

This was a four-part study. Initially, each subject was randomly assigned (2:1 ratio) to a training cohort or a validation cohort, first identifying patient-related variables in the training cohort that impacted survival time after sorafenib cessation. The multivariate analyses were performed to extract meaningful prognostic indices, and resultant $\beta$ regression coefficient of each variable was used to construct a new NEXT (Survival after Stopping Nexavar Treatment) scoring system. Third, the newly established NEXT scoring system was applied to the validation cohort to confirm its utility in properly stratifying patients by survival. Lastly, the prognostic accuracy of the NEXT score was validated in an external Korean cohort.

\section{Statistical analysis}

The interval between date of stopping sorafenib and death or last follow-up was used to calculate survival. Continuous variables were compared using the Student t-test or Mann-Whitney $\mathrm{U}$ test, and categorical variables were compared using chisquare or Fisher exact test. Variables with $\mathrm{p}<0.05$ in univariate analyses were included in multivariate analyses, and factors with $\mathrm{p}<0.05$ were ultimately selected as elements of the new formula. All of the variables significant in univariate analyses were tested using the Cox multivariate analyses. The multivariate Cox proportional hazard models were used to calculate the $\beta$ regression coefficient, $\mathrm{p}$-value, adjusted hazard ratio (HR), and its 95\% confidence interval (CI) for each of the selected risk predictors. Survival was estimated using the Kaplan-Meier method, and between-group survival differences were assessed using the log-rank test. The discriminative ability of specific prediction models for survival was determined based on receiver operating characteristic (ROC) curve analysis, expressed as the area under the ROC curve (AUROC). All of the statistical analyses relied on standard software SPSS version 21.0 (SPSS Corp., Armonk, NY, USA), with a two-sided p-value $<0.05$ considered statistically significant.

\section{RESULTS}

\section{Baseline characteristics overall and in patient subsets}

After excluding 257 ineligible candidates, a total of 409 patients with HCC who had discontinued sorafenib therapy were recruited for this study (Fig. 1). Subjects were randomly as- 
signed in a 2:1 ratio of training $(n=272)$ or validation $(n=137)$ cohorts. Baseline characteristics determined on the day of stopping sorafenib are shown in Table 1 . The median age of the entire study population (men, 342; women, 67) was 57 years. In 191 patients (46.7\%), performance status was poor (Eastern Cooperative Oncology Group [ECOG] score $\geq 2$ ), whereas 98 (24.0\%) and 120 (29.3\%) patients had ECOG scores of 1 and 0, respectively. Child-Pugh classes A, B, and C included 185 (45.2\%), 156 (38.1\%), and 68 patients (16.6\%), respectively. Etiology of HCC was hepatitis B virus (HBV) in 322 patients (78.8\%), hepatitis C virus in 17 patients (4.2\%), heavy alcohol consumption in 53 patients (13.0\%), and others in 17 patients (4.2\%). The median $\alpha$-fetoprotein (AFP) level was 467.0 (interquartile range, 9.8 to 7,129.0) ng/mL. Barcelona Clinic Liver Cancer (BCLC) stage C and D was identified in 330 (80.7\%) and 79 patients (19.3\%), respectively.

Previous treatments prior to sorafenib use were trans-arterial chemoembolization or trans-arterial chemoinfusion in 62 pa-

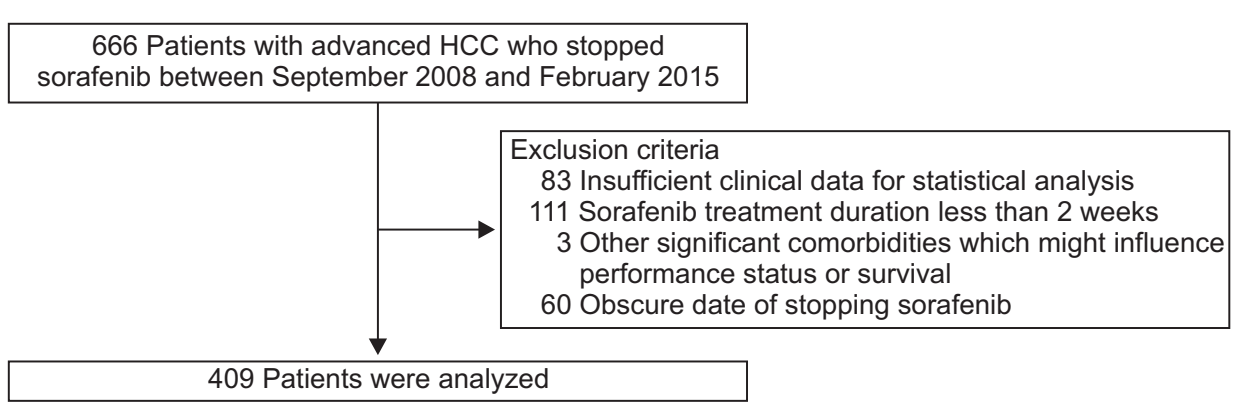

Fig. 1. Flow of study participant selection. Of 666 patients with hepatocellular carcinoma (HCC) who discontinued sorafenib between September 2008 and February 2015, 257 were excluded based on defined criteria. The final statistical analysis reflected 409 qualified patients.

Table 1. Baseline Characteristics on the Day of Stopping Sorafenib

\begin{tabular}{|c|c|c|c|c|}
\hline Variable & $\begin{array}{c}\text { All } \\
(\mathrm{n}=409)\end{array}$ & $\begin{array}{l}\text { Training cohort } \\
(\mathrm{n}=272,66.5 \%)\end{array}$ & $\begin{array}{l}\text { Validation cohort } \\
(\mathrm{n}=137,33.5 \%)\end{array}$ & p-value \\
\hline \multicolumn{5}{|l|}{ Demographic variables } \\
\hline Age, yr & $57(51-65)$ & $57(51-65)$ & $56(52-64)$ & 0.765 \\
\hline Male sex & $342(83.6)$ & $225(82.7)$ & $117(85.4)$ & 0.572 \\
\hline Viral etiology & 339 (82.9) & $222(81.6)$ & $117(85.4)$ & 0.404 \\
\hline $\mathrm{HBV}$ & $322(78.7)$ & 214 (78.6) & $108(78.8)$ & \\
\hline $\mathrm{HCV}$ & $17(4.2)$ & $11(4.0)$ & $6(4.4)$ & \\
\hline Alcohol & $53(13.0)$ & $36(13.2)$ & $17(12.4)$ & \\
\hline Others & $17(4.2)$ & $12(4.4)$ & $5(3.6)$ & \\
\hline Hypertension & $157(38.4)$ & 107 (39.3) & $50(36.5)$ & 0.592 \\
\hline Diabetes mellitus & $101(24.7)$ & $63(23.2)$ & 38 (27.7) & 0.332 \\
\hline $\mathrm{ECOG} \geq 2$ & $191(46.7)$ & $125(46.0)$ & $66(48.2)$ & 0.676 \\
\hline Child-Pugh class A & $185(45.2)$ & & & \\
\hline Child-Pugh class B-C & $224(54.8)$ & $146(53.7)$ & 78 (56.9) & 0.593 \\
\hline MELD score & $19.8(7.3-30.3)$ & $19.3(6.8-29.2)$ & $19.7(7.0-31.8)$ & 0.660 \\
\hline \multicolumn{5}{|l|}{ Laboratory variables } \\
\hline Alanine aminotransferase, IU/L & $35.0(23.0-59.5)$ & $35.5(23.3-59.0)$ & $32.0(23.0-63.5)$ & 0.263 \\
\hline Serum sodium, $\mathrm{mEq} / \mathrm{L}$ & $137(133-140)$ & $137(133-140)$ & $136(132-139)$ & 0.847 \\
\hline$\alpha$-Fetoprotein $>400 \mathrm{ng} / \mathrm{mL}$ & $209(51.1)$ & $137(50.4)$ & $72(52.6)$ & 0.753 \\
\hline Total bilirubin, mg/dL & $1.1(0.7-2.5)$ & $1.1(0.7-2.6)$ & $1.0(0.7-2.1)$ & 0.237 \\
\hline Serum albumin, $\mathrm{g} / \mathrm{dL}$ & $3.2(2.6-3.7)$ & $3.2(2.6-3.7)$ & $3.1(2.7-4.0)$ & 0.253 \\
\hline Prothrombin time, INR & $1.1(1.0-1.3)$ & $1.1(1.0-1.3)$ & $1.1(1.0-1.2)$ & 0.426 \\
\hline Serum creatinine, $\mathrm{mg} / \mathrm{dL}$ & $0.8(0.6-1.0)$ & $0.8(0.6-1.0)$ & $0.8(0.7-1.0)$ & 0.664 \\
\hline BCLC stage D & 79 (19.3) & $55(20.2)$ & $24(17.5)$ & 0.596 \\
\hline Anticancer treatment after sorafenib & $182(44.5)$ & $128(47.1)$ & $54(39.4)$ & 0.171 \\
\hline
\end{tabular}

Data are presented as median (interquartile range) or number (\%).

HBV, hepatitis B virus; HCV, hepatitis C virus; ECOG, Eastern Cooperative Oncology Group; MELD score, model for end-stage liver disease score; INR, international normalized ratio; BCLC, Barcelona Clinic Liver Cancer. 
tients (15.2\%), hepatic artery infusion chemotherapy (HAIC) in 29 (7.1\%), surgical treatment in 22 (5.4\%), radiotherapy in five (1.2\%), concurrent chemoradiotherapy in three (0.7\%), liver transplantation in two (0.5\%), radiofrequency ablation in one (0.2\%), and combined treatments in 197 patients (48.2\%). Anticancer treatment after sorafenib cessation included systemic chemotherapy in 45 patients (11.0\%), radiotherapy in 25 (6.1\%), TACE in 24 (5.9\%), HAIC in 20 (4.9\%), radiofrequency ablation in one (0.2\%), and others in 10 patients (2.4\%).

In the training cohort, median age (men, 225; women, 47) was 57 years, and the majority $(82.7 \%, n=225)$ had virus-related HCC. As defined above, poor performance status and diminished liver function applied to approximately half of the training cohort (46.0\% and 53.7\%, respectively). High tumor burden (AFP level $>400 \mathrm{ng} / \mathrm{mL}$ ) was identified in 137 patients (50.4\%), and 55 patients (20.2\%) had terminal stage HCC (BCLC D stage). In the validation cohort, median age (men, 117; women, 20) was 56 years, and none of the related baseline characteristics significantly differed from those of the training cohort ( $p>0.05$ ) (Table 1). Median duration of sorafenib treatment and median cumulative dosing in each cohort were similar (14.0 weeks in training cohort vs 12.9 weeks in validation cohort; $58,400 \mathrm{mg}$ in training cohort vs 58,400 mg in the validation cohort; all p>0.05).

\section{Survival-related prognostic factors and multivariate analyses in training cohort}

Based upon univariate analyses, an ECOG score $\geq 2$, ChildPugh class B or C, alanine aminotransferase (ALT) level $>40$ IU/L, serum sodium levels $\leq 135 \mathrm{mEq} / \mathrm{L}$, AFP level $>400 \mathrm{ng} /$ $\mathrm{mL}$, radiological progression, and 2nd-line chemotherapy after sorafenib failure were identified as significant indices of poorer survival $(p<0.05)$ (Table 2). Of these, four factors emerged as independent negative predictors of survival $(\mathrm{p}<0.05)$ based on multivariate analyses as follows: poor performance (ECOG score $\geq 2$; HR, 2.391; 95\% CI, 1.205 to 4.743); decreased liver function (Child-Pugh class B or C; HR, 2.105; 95\% CI, 1.039 to 4.266); low serum sodium level ( $\leq 135 \mathrm{mEq} / \mathrm{L}$; HR, 2.499; 95\% CI, 1.193 to 5.234); and high AFP level ( $>400 \mathrm{ng} / \mathrm{mL}$; HR, 2.329; 95\% CI, 1.287 to 4.215 ) (Table 2 ).

\section{Generating a NEXT score}

Because of the overlap with ECOG and Child-Pugh class, we excluded BCLC staging to establish the new scoring system. In the training set, four independent predictors were significant predictors for overall survival. The regression coefficient of each risk predictor from the multivariate Cox proportional hazard model was divided by the regression coefficient of the ChildPugh class, which was lowest among the four variables, and then was rounded to an integer value to generate each score respectively (Table 3). Then, we allocated 1 point for an ECOG score $\geq 2$, Child-Pugh class B or C, serum sodium level $\leq 135$ $\mathrm{mEq} / \mathrm{L}$, and AFP level $>400 \mathrm{ng} / \mathrm{mL}$ to generate new models, which showed acceptable AUROC values in predicting 1-, 3-, and 6-month survival $(0.805,0.809$, and 0.774 , respectively) (Table 3, Fig. 2). Hence, this model served as the NEXT scoring system, with a range of 0 to 4 points. When applied to the validation cohort $(\mathrm{n}=137)$, AUROC values in predicting 1-, 3-, and 6 -month survival were $0.783,0.728$, and 0.673 , respectively (p<0.05) (Fig. 2).

\section{Survival outcomes, based on risk stratification by NEXT score, in all of the patients, and patient subsets}

Median survival of all of the patients was 3.9 months (range, 0.1 to 69.1 months), and survival rates at 1-, 3-, and 6-months were $77.5 \%$, 49.1\%, and 30.1\%, respectively. When stratified by NEXT score into low-risk (NEXT score 0$)(n=85,20.8 \%)$, intermediate-risk (NEXT score 1 to 2) ( $n=202,49.4 \%$ ), and highrisk (NEXT score 3 to 4 ) groups ( $\mathrm{n}=122,29.8 \%$ ), median survival times were 7.9 months (range, 1.2 to 69.1 months), 4.0 months (range, 10.4 to 41.0 months), and 1.0 month (range, 0.1 to 12.9 months), respectively (Table 4). Survival rates at 1-, 3-, and 6-months were $100.0 \%, 83.8 \%$, and $56.3 \%$, respectively for the low-risk group; $87.0 \%, 57.0 \%$, and $35.5 \%$, respectively for the intermediate-risk group; and 48.8\%, 15.5\%, and 5.4\%, respectively for the high-risk group. Patients in the intermediate-risk $(H R, 2.8)$ and high-risk (HR, 9.9) groups had significantly greater risks of mortality, compared to those in the low-risk reference group ( $\mathrm{p}<0.001)$. Median survival and survival rates of each risk group in training and validation cohort were summarized in Table 4. There were no significant differences in overall survival between the training and validation sets after stopping sorafenib ( $p=0.848$ ) (Supplementary Fig. 1). Kaplan-Meier curves of each risk group in the training and validation cohort confirmed that survival after stopping sorafenib significantly differed among groups ( $\mathrm{p}<0.05$, log-rank test) (Fig. 3).

\section{External validation of NEXT score}

The NEXT score was validated externally $(n=80)$. The mean age of the external cohort (men, 71; women, 9) was 57 years (Supplementary Table 1). The median survival was 1.2 months (range, 0.1 to 71.8 months) for all of the patients, 13.8 months (range, 0.3 to 71.8 months) for the low-risk group, 1.6 months (range, 0.2 to 9.7 months) for the intermediate-risk group, and 0.4 months (0.1 to 5.7 months) for the high-risk group (Supplementary Table 2). The AUROC values for prediction of 1-, 3-, and 6-month survival were 0.734 (95\% CI, 0.637 to 0.831 ), 0.730 (95\% CI, 0.621 to 0.863), and 0.713 (95\% CI, 0.508 to 0.890 ), respectively $(\mathrm{p}<0.05)$.

\section{DISCUSSION}

In this study, the prognosis after cessation of sorafenib treatment in patients with HCC was poor, marked by a median survival time of only 3.9 months. Subsequently, four indepen- 
Table 2. Independent Predictors of Death in the Training Cohort $(n=272)$

\begin{tabular}{|c|c|c|c|c|}
\hline \multirow{2}{*}{ Variable } & \multirow{2}{*}{$\begin{array}{c}\text { Univariate } \\
\mathrm{p} \text {-value }\end{array}$} & \multicolumn{3}{|c|}{ Multivariate } \\
\hline & & $\mathrm{HR}$ & $95 \% \mathrm{CI}$ & p-value \\
\hline \multicolumn{5}{|l|}{ During sorafenib treatment } \\
\hline Adverse events ( $\geq$ grade 3 ) & 0.088 & - & - & - \\
\hline \multicolumn{5}{|l|}{ At the time of stopping sorafenib } \\
\hline Age, yr & 0.130 & - & - & - \\
\hline Male sex & 0.655 & - & - & - \\
\hline Viral etiology & 0.566 & - & - & - \\
\hline Hypertension & 0.363 & - & - & - \\
\hline Diabetes mellitus & 0.873 & - & - & - \\
\hline \multicolumn{5}{|l|}{ ECOG } \\
\hline $0-1$ & $<0.001$ & 1.000 & & \\
\hline$\geq 2$ & & 2.391 & $1.205-4.743$ & 0.013 \\
\hline \multicolumn{5}{|l|}{ Child-Pugh class } \\
\hline A & $<0.001$ & 1.000 & & \\
\hline $\mathrm{B}-\mathrm{C}$ & & 2.105 & $1.039-4.266$ & 0.039 \\
\hline \multicolumn{5}{|l|}{ Alanine aminotransferase, IU/L } \\
\hline$\leq 40$ & 0.001 & 1.000 & & \\
\hline$>40$ & & 1.222 & $0.668-2.235$ & 0.516 \\
\hline \multicolumn{5}{|l|}{ Serum sodium, $\mathrm{mEq} / \mathrm{L}$} \\
\hline$>135$ & $<0.001$ & 1.000 & & \\
\hline$\leq 135$ & & 2.499 & $1.193-5.234$ & 0.015 \\
\hline \multicolumn{5}{|l|}{$\alpha$-Fetoprotein, $\mathrm{ng} / \mathrm{mL}$} \\
\hline$\leq 400$ & $<0.001$ & 1.000 & & \\
\hline$>400$ & & 2.329 & $1.287-4.215$ & 0.005 \\
\hline \multicolumn{5}{|l|}{ Radiologic progression } \\
\hline No & $<0.001$ & 1.000 & & \\
\hline Yes & & 1.594 & $0.822-3.091$ & 0.168 \\
\hline The reason for sorafenib discontinuation & 0.884 & & & \\
\hline \multicolumn{5}{|l|}{ After stopping sorafenib } \\
\hline \multicolumn{5}{|l|}{ 2nd-line chemotherapy } \\
\hline Yes & 0.038 & 1.000 & & \\
\hline No & & 1.114 & $0.566-2.195$ & 0.754 \\
\hline
\end{tabular}

HR, hazard ratio; CI, confidence interval; ECOG, Eastern Cooperative Oncology Group.

Table 3. Risk Stratification According to the Score Comprising Independent Prognostic Factors (NEXT Model) and Survival Prediction in the Training Cohort

\begin{tabular}{|c|c|c|c|}
\hline Variable & $\beta$ Coefficient & $\mathrm{p}$-value & Risk scoring \\
\hline $\mathrm{ECOG} \geq 2$ & 0.872 & 0.013 & 1 \\
\hline Child-Pugh class B-C & 0.744 & 0.039 & 1 \\
\hline Serum sodium $\leq 135 \mathrm{mEq} / \mathrm{L}$ & 0.916 & 0.015 & 1 \\
\hline$\alpha$-Fetoprotein $>400 \mathrm{ng} / \mathrm{mL}$ & 0.845 & 0.005 & 1 \\
\hline Survival prediction & & AUROC (95\% CI) & \\
\hline $1 \mathrm{mo}$ & & $0.805(0.748-0.861)$ & \\
\hline $3 \mathrm{mo}$ & & $0.809(0.758-0.860)$ & \\
\hline $6 \mathrm{mo}$ & & $0.774(0.718-0.831)$ & \\
\hline
\end{tabular}

ECOG, Eastern Cooperative Oncology Group; AUROC, area under the receiver operating characteristic curve; CI, confidence interval. 
A

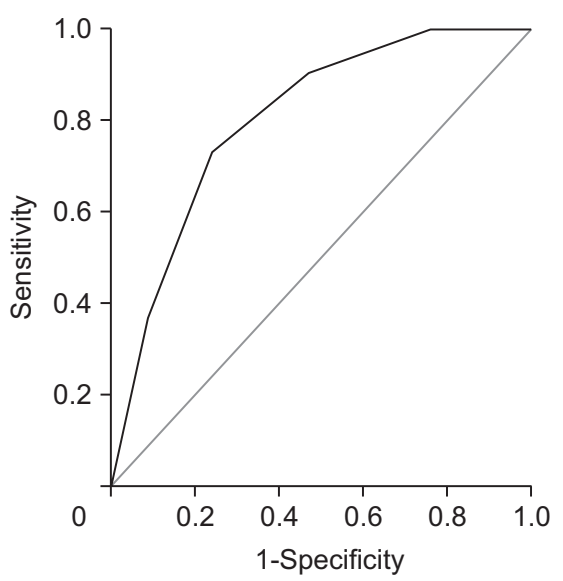

D

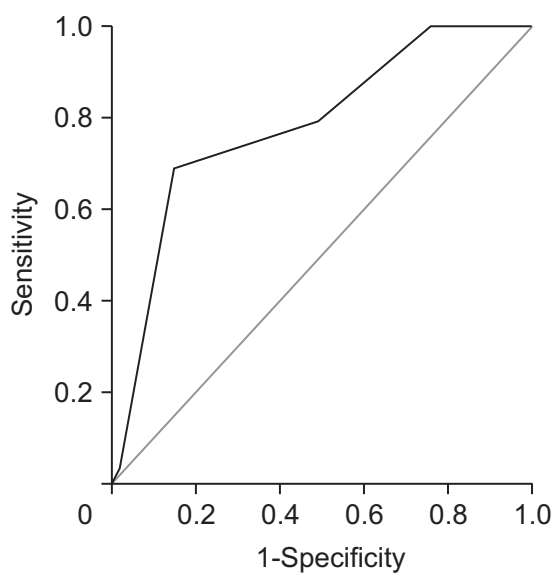

B

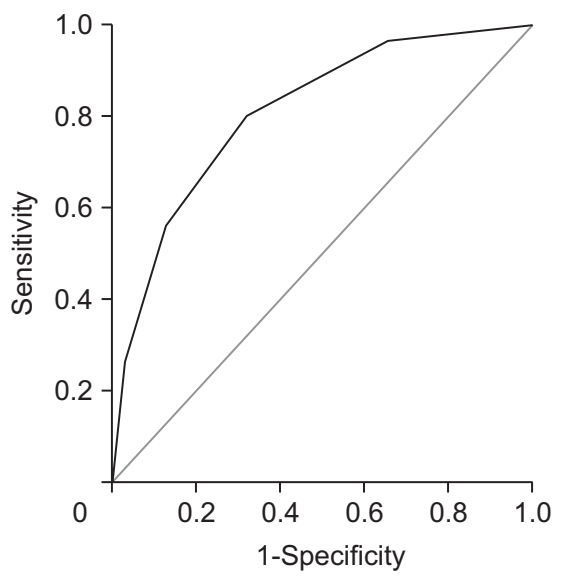

E

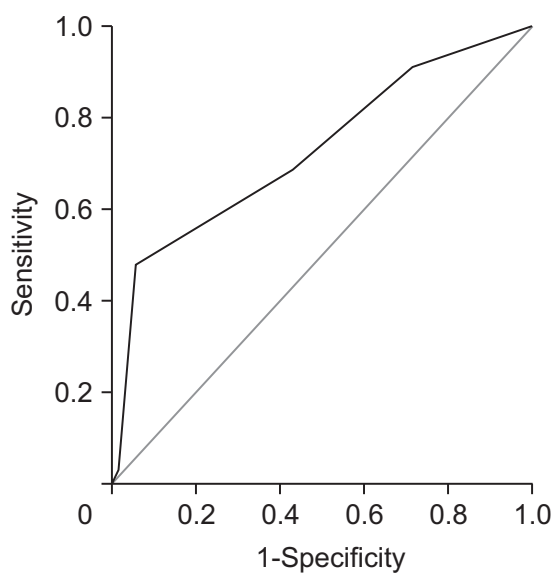

C

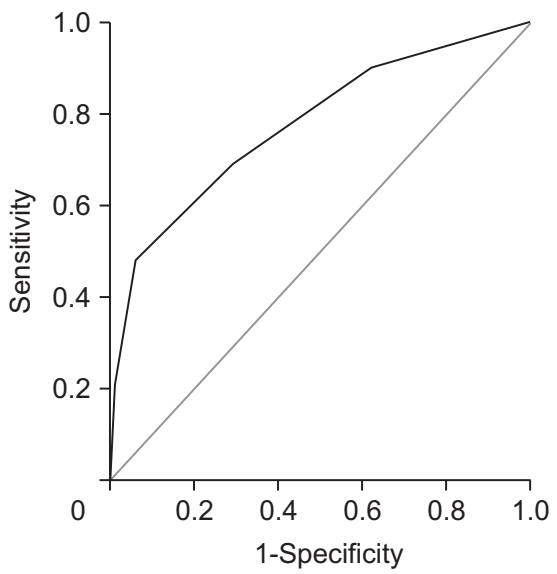

F

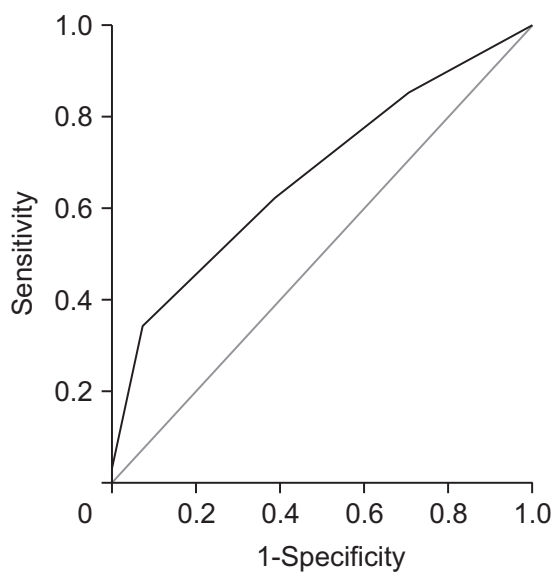

Fig. 2. Receiver operating characteristic (ROC) curves for survival prediction models at (A) 1-, (B) 3-, and (C) 6-months in the training and (D) 1-, (E) 3-, and (F) 6-months in the validation sets. Among the potential prototypes, a new model, allocating 1 point each for Eastern Cooperative Oncology Group (ECOG) $\geq 2$, Child-Pugh class B or C, serum sodium $\leq 135 \mathrm{mEq} / \mathrm{L}$, and $\alpha$-fetoprotein $>400 \mathrm{ng} / \mathrm{mL}$, showed high area under the ROC curve values and was used to generate NEXT scores (Survival after Stopping Nexavar Treatment).

Table 4. Survival Outcomes According to Risk Stratification

\begin{tabular}{|c|c|c|c|c|c|c|}
\hline \multirow{2}{*}{ Risk } & \multirow{2}{*}{ Survival, median (range), mo } & \multicolumn{3}{|c|}{ Overall survival rate, $\%$} & \multicolumn{2}{|c|}{ Cox regression } \\
\hline & & $1 \mathrm{mo}$ & $3 \mathrm{mo}$ & $6 \mathrm{mo}$ & $\mathrm{HR}(95 \% \mathrm{CI})$ & $\mathrm{p}$-value \\
\hline \multicolumn{7}{|l|}{ Training cohort } \\
\hline Low-risk $(n=50)$ & $9.2(1.2-69.1)$ & 100.0 & 90.0 & 62.0 & 1.0 & \\
\hline Intermediate-risk $(\mathrm{n}=126)$ & $4.2(0.4-41.0)$ & 86.5 & 54.8 & 36.5 & $3.5(2.1-5.9)$ & $<0.001$ \\
\hline High-risk $(n=96)$ & $1.2(0.1-12.9)$ & 52.1 & 17.7 & 5.2 & $12.6(7.63-21.8)$ & $<0.001$ \\
\hline \multicolumn{7}{|l|}{ Validation cohort } \\
\hline Low-risk $(n=35)$ & $5.8(2.4-19.0)$ & 100.0 & 73.3 & 46.7 & 1.0 & \\
\hline Intermediate-risk $(\mathrm{n}=76)$ & $4.0(2.0-6.9)$ & 87.8 & 60.8 & 33.8 & $1.9(1.1-3.3)$ & 0.031 \\
\hline High-risk $(n=26)$ & $0.8(0.1-9.4)$ & 39.4 & 9.1 & 6.1 & $6.7(3.5-12.9)$ & $<0.001$ \\
\hline \multicolumn{7}{|l|}{ All participants } \\
\hline \multicolumn{7}{|l|}{ All } \\
\hline Low-risk $(n=85)$ & $7.9(1.2-69.1)$ & 100.0 & 83.8 & 56.3 & 1.0 & \\
\hline Intermediate-risk $(\mathrm{n}=202)$ & $4.0(0.4-41.0)$ & 87.0 & 57.0 & 35.5 & $2.8(1.9-4.1)$ & $<0.001$ \\
\hline High-risk $(\mathrm{n}=122)$ & $1.0(0.1-12.9)$ & 48.8 & 15.5 & 5.4 & $9.9(6.6-15.0)$ & $<0.001$ \\
\hline
\end{tabular}

HR, hazard ratio; CI, confidence interval. 

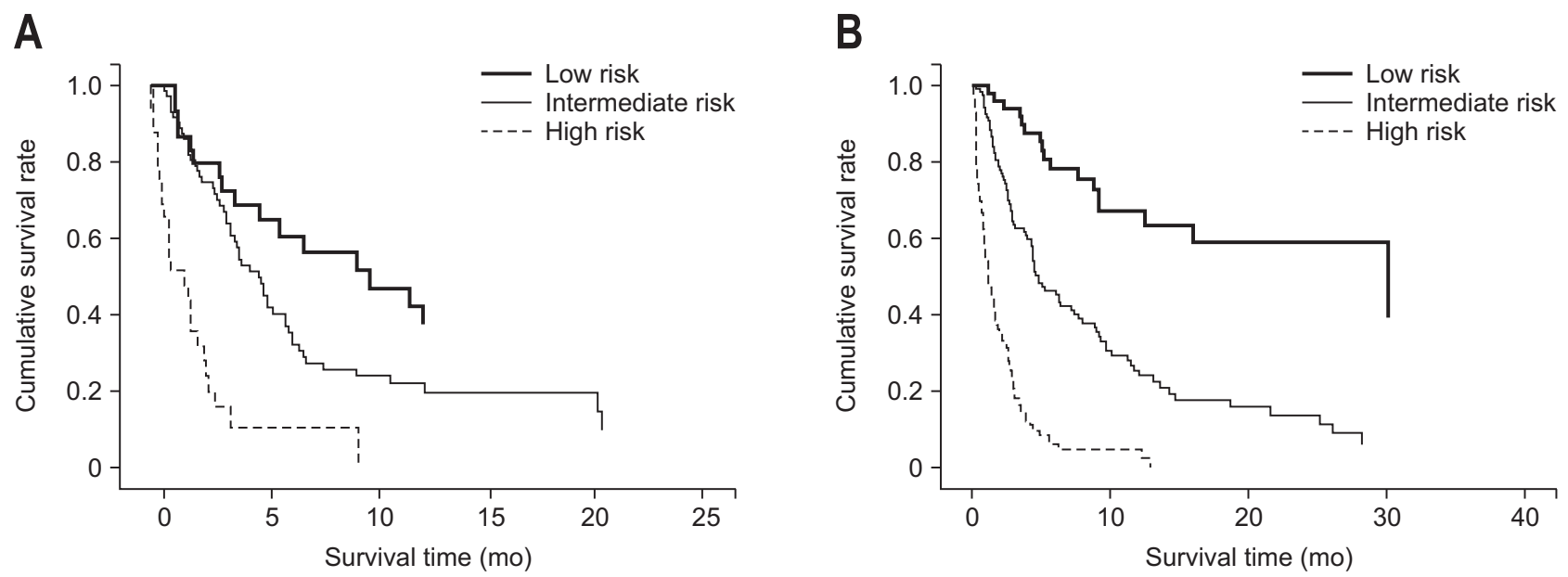

Fig. 3. Survival curves of (A) training and (B) validation cohorts based on risk stratification according to the NEXT score (Survival after Stopping Nexavar Treatment). Kaplan-Meier curves of risk groups in the training and validation cohorts showed significant differences in survival after stopping sorafenib ( $<<0.05$, log-rank test).

dent negative prognostic factors were identified in this setting, including an ECOG score $\geq 2$, Child-Pugh class B or C, serum sodium level $\leq 135 \mathrm{mEq} / \mathrm{L}$, and high AFP level $>400 \mathrm{ng} / \mathrm{mL}$. Using these factors, the NEXT scoring system was able to predict mortality. This system showed acceptable accuracy in predicting mortality at 1-, 3-, and 6-months after sorafenib treatment when applied to training (AUROC, 0.774 to 0.809 ) and validation cohorts (AUROC, 0.673 to 0.783 ). When our study population was stratified based on the NEXT score into three groups to assess the difference in survival, the median survival of low and high-risk groups significantly differed between the training (9.2 months vs 1.2 months, respectively) and validation (5.8 months vs 0.8 months, respectively) cohorts (log-rank test, $\mathrm{p}<0.05$ ). These outcomes support the heterogeneity of survival outcomes, once sorafenib is discontinued.

Our study had several strengths. First, reliable statistical power was ensured based on the relatively large sample size (>400 subjects) using the only approved first-line systemic targeted regimen (i.e., sorafenib) suitable to construct and validate a new prediction model. In a similar fashion, a recent Taiwanese study ${ }^{17}$ showed that Child-Pugh class A status or potential eligibility for clinical trials correlated with a better prognosis in patients with advanced HCC who failed first-line therapy. The potential pitfalls of that investigation were its small overall sample size (<200 subjects), a lack of adjustment for confounding prognostic influences, and the heterogeneity of first-line systemic regimens used. The latter included sorafenib ( 20\%), sunitinib, brivanib, and bevacizumab, among others. Because sorafenib is the only targeted agent currently approved in this context, the clinical applicability of this Taiwanese study may be limited.

Other studies have reported survival outcomes (median progression-free survival, 4.6 to 9.85 months) after discontinuing sorafenib and have identified independent predictive variables.
However, only patients with BCLC C stage HCC were recruited, ${ }^{18}$ and patient sampling was sparse $(\mathrm{n}=85)$ despite limiting enrollees to sorafenib-treated patients. ${ }^{15}$ Another distinct advantage is the simplicity of generating a NEXT score. The required determinants are readily available as routine laboratory tests and clinical data, making this predictive model easy to apply in routine clinical practice. Because other serum biomarkers, such as Ang2, can be assayed before starting sorafenib, revision of the NEXT score is possible based on future studies.

The NEXT score is generated from four prognostic factors carrying similar mortality risk. ECOG score and Child-Pugh class are well-known prognostic indices, even beyond this therapeutic context. ${ }^{11,19,20}$ Child-Pugh score, a "robust" predictor of mortality, has been the standard for ranking patient status in cirrhosis for nearly three decades and in assessing end-stage liver disease. ${ }^{21,22}$ In a prior study by Shao et al., ${ }^{17} 36 \%$ of patients with preserved liver function and 11\% of patients with good performance status at baseline eventually deteriorated to ChildPugh class B or C and an ECOG score $\geq 2$ after sorafenib treatment. Thus, our rationale to re-assess these two pivotal factors at the time of stopping sorafenib is valid. Low serum sodium levels, which also served as one of our independent predictors, is associated with severe complications, including ascites, hepatorenal syndrome, and even death. ${ }^{23}$ Thus, it is not surprising that hyponatremia is one of our independent prognostic factors. A rise in tumor marker levels is suggestive of high HCC burden and malignant potential, whether or not morphologic progression is evident. ${ }^{24,25}$ Hence, an unfavorable prognosis was associated with heightened AFP levels in our cohort. However, the reason for sorafenib discontinuation did not show statistical significance $(p=0.884)$; thus, we excluded this factor from the NEXT score.

Several limitations of this study are acknowledged, the first being its retrospective design. Potential bias in decisions to stop 
sorafenib treatment, particularly in the event of intolerance, may have influenced survival. In addition, the small number of patients in the validation group $(n=80)$ compared to the number of patients in the training group $(n=272)$ may have influenced the results. Next, second-line anticancer treatments after stopping sorafenib were given to approximately half of our patients (44.5\%), as directed by physicians. Although we explored whether second-line anticancer treatment after sorafenib failure was prognostic, it was only significant in univariate analyses, probably due to the extremely short survival duration of the study population and the heterogeneity of second-line regimens. Thus, further prospective studies are required to resolve this issue. Third, because HBV is the most frequent etiology of HCC in Korea, different from Western countries, the NEXT score was externally validated using a second Korean cohort. For more widespread use of the NEXT score, validation studies in Western cohorts should be performed. Fourth, the mRECIST criteria have not been fully validated as response-assessment criteria during sorafenib treatment. ${ }^{26}$ Lastly, in our study, we calculated the NEXT score based on clinical data obtained on the day of stopping sorafenib. Because the NEXT score was based on the binary categorization of four prognostic variables, few patients experienced improvement in the NEXT score during the 2 weeks after stopping sorafenib, and even fewer were re-classified into other risk groups. Although we believe that the time restriction may prevent further bias, further studies are required to address this issue.

In conclusion, we found that survival after discontinuing sorafenib was poor in patients with HCC. We also validated a newly developed NEXT scoring system to predict survival in this setting, based on four prognostic factors identified in our study population, which may be used to predict survival in patients whose sorafenib treatment is discontinued. Although further validation studies are required, the NEXT score may have merit in a second-line chemotherapeutic scenario, providing a basis for decisions on further treatment.

\section{CONFLICTS OF INTEREST}

No potential conflict of interest relevant to this article was reported.

\section{ACKNOWLEDGEMENTS}

We thank Dongsoo Jang (Yonsei University) for technical assistance on the preparation of figures.

Author contributions: Study conception, S.U.K. and D.Y.K. Study design, H.S.K., H.W.L., S.U.K., and D.Y.K. Participation in patient management and data collection, M.Y.J., J.Y.H., S.Y.P., Y.R.L., S.K.J., S.H.L., S.Y.J., B.K.K., J.Y.P., D.Y.K., S.H.A., W.Y.T., and K.H.H. Contribution to the data acquisition, responsibility for writing the paper, and statistical analysis, H.S.K., H.W.L.,
S.U.K. and D.Y.K. All authors reviewed the paper and approved the final version.

\section{REFERENCES}

1. Jemal A, Bray F, Center MM, Ferlay J, Ward E, Forman D. Global cancer statistics. CA Cancer J Clin 2011;61:69-90.

2. Forner A, Llovet JM, Bruix J. Hepatocellular carcinoma. Lancet 2012;379:1245-1255.

3. Chang YS, Adnane J, Trail PA, et al. Sorafenib (BAY 43-9006) inhibits tumor growth and vascularization and induces tumor apoptosis and hypoxia in RCC xenograft models. Cancer Chemother Pharmacol 2007;59:561-574.

4. Wilhelm SM, Carter C, Tang L, et al. BAY 43-9006 exhibits broad spectrum oral antitumor activity and targets the RAF/MEK/ERK pathway and receptor tyrosine kinases involved in tumor progression and angiogenesis. Cancer Res 2004;64:7099-7109.

5. Cheng AL, Kang YK, Chen Z, et al. Efficacy and safety of sorafenib in patients in the Asia-Pacific region with advanced hepatocellular carcinoma: a phase III randomised, double-blind, placebocontrolled trial. Lancet Oncol 2009;10:25-34.

6. Llovet JM, Ricci S, Mazzaferro V, et al. Sorafenib in advanced hepatocellular carcinoma. N Engl J Med 2008;359:378-390.

7. Di Marco V, De Vita F, Koskinas J, Semela D, Toniutto P, Verslype C. Sorafenib: from literature to clinical practice. Ann Oncol 2013;24 Suppl 2:ii30-ii37.

8. Lee JM, Jang BK, Lee YJ, et al. Survival outcomes of hepatic resection compared with transarterial chemoembolization or sorafenib for hepatocellular carcinoma with portal vein tumor thrombosis. Clin Mol Hepatol 2016;22:160-167.

9. Park JG. Long-term outcomes of patients with advanced hepatocellular carcinoma who achieved complete remission after sorafenib therapy. Clin Mol Hepatol 2015;21:287-294.

10. Woo HY, Heo J. New perspectives on the management of hepatocellular carcinoma with portal vein thrombosis. Clin Mol Hepatol 2015;21:115-121

11. Lee S, Kim BK, Kim SU, et al. Clinical outcomes and prognostic factors of patients with advanced hepatocellular carcinoma treated with sorafenib as first-line therapy: a Korean multicenter study. J Gastroenterol Hepatol 2014;29:1463-1469.

12. Lee S, Kim BK, Kim SU, et al. Efficacy of sorafenib monotherapy versus sorafenib-based loco-regional treatments in advanced hepatocellular carcinoma. PLoS One 2013;8:e77240.

13. Llovet JM, Decaens T, Raoul JL, et al. Brivanib in patients with advanced hepatocellular carcinoma who were intolerant to sorafenib or for whom sorafenib failed: results from the randomized phase III BRISK-PS study. J Clin Oncol 2013;31:3509-3516.

14. Iavarone M, Cabibbo G, Biolato M, et al. Predictors of survival in patients with advanced hepatocellular carcinoma who permanently discontinued sorafenib. Hepatology 2015;62:784-791.

15. Reig M, Rimola J, Torres F, et al. Postprogression survival of patients with advanced hepatocellular carcinoma: rationale for 
second-line trial design. Hepatology 2013;58:2023-2031.

16. European Association for the Study of the Liver; European Organisation for Research and Treatment of Cancer. EASL-EORTC clinical practice guidelines: management of hepatocellular carcinoma. J Hepatol 2012;56:908-943.

17. Shao YY, Wu CH, Lu LC, et al. Prognosis of patients with advanced hepatocellular carcinoma who failed first-line systemic therapy. J Hepatol 2014;60:313-318.

18. Lee IC, Chen YT, Chao Y, et al. Determinants of survival after sorafenib failure in patients with BCLC-C hepatocellular carcinoma in real-world practice. Medicine (Baltimore) 2015;94:e688.

19. Kim HY, Park JW, Joo J, et al. Worse outcome of sorafenib therapy associated with ascites and Child-Pugh score in advanced hepatocellular carcinoma. J Gastroenterol Hepatol 2013;28:17561761.

20. Sohn W, Paik YH, Cho JY, et al. Sorafenib therapy for hepatocellular carcinoma with extrahepatic spread: treatment outcome and prognostic factors. J Hepatol 2015;62:1112-1121.

21. D’Amico G, De Franchis R; Cooperative Study Group. Upper digestive bleeding in cirrhosis: post-therapeutic outcome and prognos- tic indicators. Hepatology 2003;38:599-612.

22. D’Amico G, Garcia-Tsao G, Pagliaro L. Natural history and prognostic indicators of survival in cirrhosis: a systematic review of 118 studies. J Hepatol 2006;44:217-231.

23. Albillos A, Colombato LA, Groszmann RJ. Vasodilatation and sodium retention in prehepatic portal hypertension. Gastroenterology 1992;102:931-935.

24. Yamamoto K, Imamura H, Matsuyama Y, et al. Significance of alpha-fetoprotein and des-gamma-carboxy prothrombin in patients with hepatocellular carcinoma undergoing hepatectomy. Ann Surg Oncol 2009;16:2795-2804.

25. Yamanaka J, Yamanaka N, Nakasho K, et al. Clinicopathologic analysis of stage II-III hepatocellular carcinoma showing early massive recurrence after liver resection. J Gastroenterol Hepatol 2000;15:1192-1198.

26. Ronot M, Bouattour M, Wassermann J, et al. Alternative response criteria (Choi, European Association for the Study of the Liver, and modified Response Evaluation Criteria in Solid Tumors [RECIST]) versus RECIST 1.1 in patients with advanced hepatocellular carcinoma treated with sorafenib. Oncologist 2014;19:394-402. 